\title{
Study of the composition of the ruminal cellulolytic flora of bison and deer with rRNA-targeted oligonucleotide probes
}

\author{
L Millet, G Fonty, P Gouet
}

INRA, Laboratoire de Microbiologie, CR Clermont-Ferrand-Theix, 63122 St-Genès-Champanelle, France

The molecular phylogenetic framework provided by comparative rRNA sequencing has given a basis for describing the community structure of natural anaerobic ecosystems such as the rumen and for studying the ecology of microbial populations without the limitations imposed by traditional culture-based enumeration, pure culture-isolation and biochemical identification. The aim of this work was to investigate the qualitative and quantitative composition of the ruminal cellulolytic flora of bisons and deers by oligonucleotide probe hybridization.

Animals were fed a forage-based diet. Rumen samples (25 to $30 \mathrm{~g}$ of liquid and solid phases) were taken from 3 bisons and 2 deers just after slaughtering, immediately frozen at $-80^{\circ} \mathrm{C}$, freeze-dried and finely ground. RNAs were extracted from $50 \mathrm{mg}$ of freeze-dried rumen contents after mechanical disruption of the microorganisms with zirconium beads (Stahl et al, 1988, Appl Environ Microbiol, 1079-1084).

The following ${ }^{32} \mathrm{P}$-labelled oligonucleotide probes (specific of the 16S rRNA) were used: the universal probe EUB 338 targeting the bacterial domain (Stahl and Amann, 1991, In Sequencing and hybridization techniques in bacterial systematics, Stackebrandt and Goodfellow eds, 205-248), probe F650 targeting Fibrobacter succinogenes (Amann et al, 1990, J Bacteriol, 172, 762-770), probe RAL 196 and probe RFL 1269 targeting Ruminococcus albus and $R$. flavefaciens, respectively (Odenyo et al, 1994, Appl Environ
Microbiol, 60, 3688-3696).

The techniques used for labelling the probes, slot-blot hybridization and quantification of bound probes were performed as described by Stahl et al (1988, Appl Environ Microbiol, 10791084). The abundances of each bacterial cellulolytic species are expressed as percentages of the total bacterial 16S rRNA in the sample. The total 16S rRNA was quantified with the universal probe EUB 338 in combination with RNA standards.

The 3 targeted-bacterial species were present in the rumen of the five animals. $F$. succinogenes was the predominant species in both bisons and deers, but the percentages of rRNA of this species were higher in bisons than in deers. The percentages of rRNA of $R$. flavefaciens were also higher in bisons than in deers while those of $R$. albus were higher in deers than in bisons. In deers, population of the two ruminococci reached approximately the same level. Compare to the total bacterial population, the respective percentages of the 3 cellulolytic species are compatible with those generally obtained by cultural-based methods in the rumen of domesticated animals fed with forages.

This work, the first study of the ruminal bacterial cellulolytic population using oligonucleotide probes, demonstrates the potential of usefulness of rRNA-based techniques for studying quantitatively and qualitatively the complex microbial communities of the rumen.

Oligonucleotide probes and

$\%$ of specific $16 \mathrm{~S}$ rRNA/total bacterial $16 \mathrm{~S}$ rRNA

\begin{tabular}{|c|c|c|c|c|c|}
\hline target bacterial species & Bison 1 & Bison 2 & Bison 3 & Deer 1 & Deer 2 \\
\hline F650 & & & & & \\
\hline F. succinogenes & 30.2 & 22.0 & 33.0 & 14.3 & 21.5 \\
\hline RFL1269 & & & & & \\
\hline R. flavefaciens & 24.4 & 17.0 & 20.9 & 11.1 & 15.4 \\
\hline RAL196 & & & & & \\
\hline R. albus & 9.3 & 8.3 & 5.8 & 12.0 & 14.0 \\
\hline
\end{tabular}

